\title{
Transport properties of olivine grain boundaries from electrical conductivity experiments
}

\author{
Anne Pommiera, David L. Kohlstedtb, Lars N. Hansenc, \\ Stephen Mackwelld ${ }^{*}$, Miki Tasakab ${ }^{b}$ Florian Heidelbache, \\ Kurt Leinenweberf
}

a University of California San Diego, Scripps Institution of Oceanography, La Jolla, CA 92093, USA

b Department of Earth Sciences, University of Minnesota, Minneapolis, MN 55455, USA

c University of Oxford, Department of Earth Sciences, ? ? South Parks Road, ?0xford OX1 3AN, United Kingdom

d Lunar and Planetary Institute, Universities Space Research Association, Houston, Texas 77058, USA

e Bayerisches GeoInstitut, University of Bayreuth, Germany

f School of Molecular Sciences, Arizona State University, Tempe, AZ 85287-1604, USA?

* Corresponding author. Email: mackwell@lpi.usra.edu 
Abstract Grain boundary processes contribute significantly to electronic and ionic transport in materials within Earth's interior. We report a novel experimental study of grain boundary conductivity in highly strained olivine aggregates that demonstrates the importance of misorientation angle between adjacent grains on aggregate transport properties. We performed electrical conductivity measurements of melt-free polycrystalline olivine (Fo90) samples that had been previously deformed at $1200^{\circ} \mathrm{C}$ and $0.3 \mathrm{GPa}$ to shear strains up to $\gamma=7.3$. The electrical conductivity and anisotropy were measured at $2.8 \mathrm{GPa}$ over the temperature range $700^{\circ}-1400^{\circ} \mathrm{C}$. We observed that (i) the electrical conductivity of samples with a small grain size (3-6 $\mu \mathrm{m})$ and strong crystallographic preferred orientation produced by dynamic recrystallization during large-strain shear deformation is a factor of 10 or more larger than that measured on coarser grained samples, (ii) the sample deformed to the highest strain is the most conductive even though it does not have the smallest grain size, and (iii) conductivity is up to a factor of $\sim 4$ larger in the direction of shear than normal to the shear plane. Based on these results combined with electrical conductivity data for coarser grained, polycrystalline olivine and for single crystals, we propose that the electrical conductivity of our fine-grained samples is dominated by grain boundary paths. In addition, the electrical anisotropy results from preferential alignment of higherconductivity grain boundaries associated with the development of a strong crystallographic preferred orientation of the grains. 
Keywords: grain boundaries; olivine; shear strain; electrical conductivity; electrical anisotropy

\section{Introduction}

Experimental studies of kinetic processes in rocks that are appropriate to various environments in Earth's interior are critical for interpreting geophysical data in terms of process. For example, anisotropy in seismic velocities measured in the oceanic lithosphere was explained as being due to the microstructural texturing (e.g., crystallographic preferred alignment of olivine grains) of olivine-rich rocks that results from shear deformation between the stiffer lithosphere and weaker underlying mantle. This interpretation was only possible due to experimental studies of shear deformation in olivine aggregates, which showed that intracrystalline deformation processes in olivine resulted in the crystallographic preferential orientation (CPO) of olivine [100] axes parallel to the direction of shear.

Experimental and computational studies have demonstrated that rocks deformed to high shear strains not only develop strong crystallographic preferred orientations (e.g., Bystricky et al. 2000; Tommasi et al. 2000; Hansen et al. 2014) but also significant grain boundary character distributions (Marquardt et al. 2015; Wagner et al. 2016). The reorientation of mineral grains during deformation tends to align the preferential crystalline shear planes in the direction of shear, which results in realignment of grain boundaries and evolution in grain boundary misorientation angles. As grain boundaries are regions of high defect densities, such changes might be expected to influence grain boundary transport processes. Thus, microstructural 
evolution resulting from shear deformation can have significant impact on the physical and mechanical properties of mantle rocks (e.g., viscosity, seismic velocity and attenuation, and electrical conductivity, as well as anisotropy of each) through the preferential alignment of not only mineral grains but also grain boundaries.

Grain boundaries are fast diffusion pathways in polycrystalline materials, with diffusivities that can be orders of magnitude faster than through mineral grains (for a recent review, see Dohmen and Milke 2010). As noted by these authors, bulk transport (flux) of chemical species through a polycrystalline aggregate is not just dependent on the mobility of the diffusing species but also the concentration of these species along the diffusion path. The same argument is true for intrinsic and impurity ionic as well as electronic conductivity in polycrystalline materials; the bulk conductivity is dependent on both the concentration and the mobility of the ionic/electronic species on the fastest transport path. While grain boundaries do provide fast pathways for ionic (e.g., Watson 2002; Hayden and Watson 2006) as well as electronic species (e.g., Roberts and Tyburczy 1991), the much higher concentrations of electronic charge carriers in grain boundaries in olivine aggregates relative to impurity ionic species (with the possible exception of protons) should generally result in electronic conduction dominating ionic conduction along grain boundary pathways.

While prior electrical conductivity studies on dry olivine aggregates have focused mainly on untextured samples that lack a CPO (e.g., Duba and Constable 1993; Roberts and Tyburczy 1991; Du Frane et al. 2005; Dai et al. 2008; 2010) or on samples containing water (e.g., Yoshino et al. 2006) or melt (e.g., Roberts and Tyburczy 1999; 
Zhang et al. 2014), there has been little systematic work looking at conductivity in dry olivine aggregates that had been deformed to high shear strains. In this study, we used electrical conductivity measurements of pre-textured samples to assess the influence of grain interior and grain boundary alignment on electronic transport. Such measurements not only provide important new information on fundamental transport properties of grain boundaries, but also allow more robust interpretation of geophysical measurements of the deep Earth.

\section{Experimental and analytical methods}

\section{Starting materials}

Our polycrystalline samples were fabricated from hot-pressed powders of San Carlos olivine of composition $\left(\mathrm{Mg}_{0.9}, \mathrm{Fe}_{0.1}\right)_{2} \mathrm{SiO}_{4}(\mathrm{Fog0})$ that had been deformed either in uniaxial compression to an axial strain $(\varepsilon)$ of 0.2 (Hansen et al. 2011) or in torsion to shear strains $(\gamma)$ of $1.3,3.5$, and 7.3 at a confining pressure $(P)$ of $0.3 \mathrm{GPa}$ and a temperature $(T)$ of $1200^{\circ} \mathrm{C}$ in a gas-medium apparatus following the procedure described by Hansen et al. (2012). The strain rate, stress, and total strain for each sample are summarized in Table 1; also included in this table is the mean grain size, which varied from 3.1 to $15 \mu \mathrm{m}$, depending on the sample. Pommier et al. (2015) previously investigated the conductivity of the sample (PT0264) deformed to $\gamma=3.5$ in one direction over a restricted temperature range.

For the torsion samples, specimens for electrical conductivity measurements were extracted from near the outer edge of the samples, that is, the region that had experienced maximum shear strain. These specimens were placed into a conductivity 
cell in a multi-anvil apparatus for electrical measurements (Pommier et al. 2015). Anisotropy in electrical conductivity was determined using measurements in three orthogonal directions for the sample deformed to the highest shear strain (PT0949) - in the shear direction, normal to shear direction in the shear plane, and normal to the shear plane. For the other samples, measurements were only made along the first two directions.

The conductivity cell in the multi-anvil apparatus for electrical measurements at 2.8 GPa followed the experimental protocol detailed in Pommier et al. (2015). In order to better assess the effect of shear deformation on electrical conductivity, we include the results from two conductivity experiments performed on polycrystalline samples of Fo90 derived from a sample deformed in compression to an axial strain of $\varepsilon=0.2$ by Hansen et al. (2011) and described in Pommier et al. (2015) (sample PI1543, Table 1). For this sample, the experiments measured conductivity parallel to and normal to the compression axis.

\section{High-pressure cell assembly}

The electrical conductivity experiments on olivine aggregates were performed at $2.8 \mathrm{GPa}$ in the multi-anvil apparatus using tungsten carbide cubes with a cornertruncation edge length of $8 \mathrm{~mm}$ in $\mathrm{MgO}$ octahedral pressure media with an edge length of $14 \mathrm{~mm}$, as illustrated in Figure 1a. Graphite cylinders or rhenium foil were used as heaters and were placed inside an outer zirconia sleeve, which provides thermal insulation. No difference in electrical conductivity measurements was observed with the two furnace types, as shown in Figure 1b. Samples used in the 
conductivity experiments were 1.0 to $1.5 \mathrm{~mm}$ in length, square in cross section (1.6 mm edge length), and were placed at the center of the cylindrical heater inside an MgO sleeve. Electrodes consisted of two molybdenum foil squares (1.6 mm edge length). The temperature was monitored with a $W_{95} R_{5}-W_{74} R_{26}$ (C-type) thermocouple inserted within a 4-bore $\mathrm{MgO}$ sleeve with the junction in contact with the top of one of the molybdenum foils. All $\mathrm{MgO}$ parts had been previously fired at $1400^{\circ} \mathrm{C}$ at room pressure for one hour and were stored in a desiccator until used for the experiments.

\section{Electrical measurements and data reduction}

Impedance spectroscopy measurements were conducted using a 2-electrode method (see Pommier et al. (2015) for details regarding the conductivity cell calibration). The conductivity cell in Figure 1a was connected to a 1260 Solartron Impedance/Gain-Phase Analyzer for electrical impedance measurements in the frequency range 1 to $5 \mathrm{MHz}$ with an applied voltage of $1 \mathrm{~V}$. The cell assembly was compressed to the target pressure of $2.8 \mathrm{GPa}$ before temperature was increased in about $20 \mathrm{~min}$ to the maximum temperature. Electrical measurements were performed during cooling, with only a few impedance spectra collected during heating. For every run, a few electrical measurements during a second heating were performed to verify the reproducibility of the measurements. All samples were quenched after the lowest temperature measurement by shutting off the power supply to the furnace before decompression. 
Electrical measurements consisted of recording the complex impedance $(Z)$ of the sample over the considered frequency range. For our samples, the real part of the complex impedance, $\operatorname{Re}(Z)$, corresponds to the electrical resistance $R$ of the sample (e.g., Huebner and Dillenburg, 1995). Because the contribution of the electrodes to the measured impedance is small compared to the contribution of the sample, the electrical conductivity of the sample, $\sigma$, can be deduced from the value of $R$ by using the following relation:

$$
\sigma=\frac{1}{R G}
$$

where the geometric factor $(G)$ is equal to the ratio of the surface area of the molybdenum electrode to the thickness of the sample. For each measurement, the electrical response of the sample was directly observed in the $(\operatorname{Re}(Z), \operatorname{Im}(Z))$ plane, and the value of $R$ was obtained from the intersection of the response of the sample with the real impedance axis. Examples of impedance spectra are presented in Figure $1 c$.

\section{Analytical observations}

Our starting materials and selected samples retrieved after the electrical experiments were characterized using electron backscatter diffraction (EBSD) analyses at UMN, Oxford, and BGI to determine the crystallographic preferred orientation (CPO) and grain size. Grain boundaries were defined as having misorientation angles $>10^{\circ}$, and the mean grain size was estimated by the linearintercept method with a scaling factor of 1.5 (Underwood 1970, pp. 80-93). In addition, post-experiment samples were analyzed by Fourier transform infrared 
(FTIR) spectroscopy at UMN to estimate the water concentration. Spectra were collected over the wavenumber range $700-8000 \mathrm{~cm}^{-1}$ with a resolution of $2 \mathrm{~cm}^{-1}$ and accumulated during 512 scans on doubly-polished sections prepared from each polycrystalline olivine sample. Longitudinal sections of samples recovered after the experiments were mounted in epoxy resin and polished to characterize (1) the sample texture and (2) the sample chemistry. The latter allowed examination of possible chemical contamination from contiguous cell parts during the experiment. Backscattered electron images, chemical composition maps, and quantitative chemical analyses were obtained with a five spectrometer JEOL JXA-8530F electron microprobe using wavelength dispersive spectrometry (WDS) and energy dispersive spectrometry (EDS) at the electron microprobe facility at ASU. SEM images were also collected using the SEM facility at BGI and using a Hitachi SU8230 FEG SEM in the Characterization Facility at UMN.

\section{Results}

\section{Microstructure and chemistry}

The textures of the four starting materials are illustrated with EBSD maps in Figure 2a. The grain size is larger and, therefore, the density of grain boundaries is smaller in the low-strain compression sample (PI1543) than in the samples deformed to higher strains in torsion. Also, the grains are slightly elongated normal to the compression direction of sample PI1543 and subparallel to the shear direction for the three samples deformed in torsion, as observed in Figure 2a,b. Textures in PI1543, 
analyzed by following grain boundary paths from one side of the sample to the other, exhibited no significant difference in path length parallel to and normal to shear.

The crystallographic preferred orientations, illustrated with the pole figures in Figure 3, demonstrate that crystallographic alignment anisotropy increases with increasing strain. The low-strain compression sample (PI1543) exhibits a weak alignment of the [010] poles parallel to the compression axis with diffuse maxima of [100] and [001] poles perpendicular to it. At the lowest shear strain in torsion (PT0941), [100] poles form two maxima, one in the shear direction and the other approximately normal to the shear plane. [010] poles display an inclined girdle distribution, whereas (001) poles form two weaker maxima at low and high angle to the shear direction. At medium and high shear strains (PT0264, PT0949), clear maxima emerge with [100] poles in the shear direction, [010] poles normal to the shear plane, and [001] poles in the shear plane, perpendicular to the shear direction. The observed texture development is consistent with previous experimental studies of polycrystalline olivine deformed in torsion (Bystricky et al. 2000; Hansen et al. 2014) as well as naturally occurring fabrics of olivine from the upper mantle (e.g., Ben Ismail and Mainprice 1998).

Comparison of the SEM images in Figure 4 of samples after the conductivity experiments with the EBSD maps in Figure 2 obtained before the conductivity runs, combined with a comparison of pole figures of our samples before and after conductivity tests (Figures 3 and 5), indicate that the grain size and crystallographic preferred orientation of the sheared samples were not significantly affected by the high-pressure, high-temperature conditions of our conductivity experiments. Any 
effects of deformation during pressurization and/or of recovery during the conductivity experiments were minimal. This result is consistent with previous work on sheared materials at similar pressure-temperature conditions (Pommier et al. 2015a; Boneh et al. 2017).

High-resolution, field-emission gun SEM observations reveal $<0.1$ vol.\% of melt in the samples used in the current study, as demonstrated in Figure 4a. Melt is present in fewer than 1 in 10 triple junctions and thus does not form an interconnected network.

As illustrated in Figure 6, FTIR analyses performed after the electrical experiments yielded water contents in the samples of $<150 \mathrm{ppm} \mathrm{H} / \mathrm{Si}$, a negligible amount compared to the solubility level of about $10,000 \mathrm{ppm} \mathrm{H/Si}$ in olivine (Kohlstedt et al. 1996) at 2.8 GPa. Electron microprobe analyses on selected samples after electrical measurements, which are presented in Table 2, demonstrate the absence of chemical contamination of the samples during electrical measurements.

\section{Electrical conductivity and anisotropy}

In all our experiments, the electrical response of the sample consists of one arc in the complex plane $\left(Z^{\prime} ; Z^{\prime \prime}\right)$, as illustrated in Figure 1c. The single semi-circle corresponds to the bulk complex impedance of the sample, including both grain interior and grain boundary contributions, as previously observed for olivine (e.g., ten Grotenhuis et al. 2004). Electrical conductivity data are presented in Figure 7 and Table 3. 
As expected, electrical conductivity increases with increasing temperature for all samples. On the Arrhenius plot in Figure 7a and in Table 3, the dependence of conductivity on temperature is not linear for the sheared samples, with a change in slope at $\sim 850^{\circ} \mathrm{C}$. The reason for this change in slope is not immediately clear, but could be due to either a change in conduction mechanism or a lack of equilibration of samples on the timescales of the measurements. For the purposes of this study, we have focused on the higher temperature data. The conductivities for all samples at temperatures above $850^{\circ} \mathrm{C}$ were fit with the Arrhenius equation

$$
\sigma=\sigma_{0} \exp \left[\frac{-H_{\mathrm{a}}}{\mathrm{R} T}\right]
$$

where $\sigma_{0}$ is the pre-exponential term, $H_{\mathrm{a}}$ the activation enthalpy, and $\mathrm{R}$ the gas constant. Values for activation enthalpy determined from our experimental results range from 85 to $140 \mathrm{~kJ} / \mathrm{mol}$ (Table 3).

Importantly, for the samples deformed in torsion, electrical conductivity is higher parallel to the shear direction than perpendicular to it, as is apparent in Figure 7a. For the sample deformed to an outer radius shear strain of $\gamma=7.3$, the anisotropy in electrical conductivity is a factor of about 1.5 in the shear plane (the ratio of conductivities between orientations 2 and 3, Figure 7a). For this sample, the electrical anisotropy indicates that transport is a factor of about 4 greater in the shear direction than normal to the shear plane (the ratio of conductivities between orientations 2 and 1, Figure 7a).

The sample deformed in compression (PI1543) showed anisotropy similar to that for the torsion samples, although the conductivity is significantly lower. In addition, the activation enthalpies are lower than for the torsion samples (Table 3). 


\section{Discussion}

Comparison of our data on electrical conductivity of deformed, polycrystalline olivine with results from previous investigations can provide important insight into the contribution of grain boundaries to electrical conductivity of olivine-rich rocks. Such a comparison is complicated, however, by the fact that electrical properties are sensitive not only to temperature and pressure but also to grain size, water (hydrogen) content, melt fraction, and oxygen fugacity (e.g., Dai et al. 2008; 2010; Gardes et al. 2014; Poe et al. 2010; ten Grotenhuis et al. 2004; Yoshino 2010; Yoshino et al. 2006; Xu et al. 2010; Zhang et al. 2014). Melt fractions at the 0.1 vol. \% level are often not detected in experiments on polycrystalline samples, and water concentrations are generally significant or undetermined in the high-pressure conductivity experiments in solid-medium apparatuses. Such observations make it difficult to make direct comparisons with our data. Therefore, for the reasons discussed in the following paragraphs, we focus on comparison of our results with extrapolation to appropriate conditions of those reported by Schock et al. (1989) on olivine single crystals, which were obtained under dry one-atmosphere conditions at well-determined oxygen fugacities.

Water: Hydrogen, an important point defect in olivine, significantly increases electrical conductivity (e.g., Karato 1990; Yoshino 2010). In solid-medium highpressure experiments, it is difficult to work at fully anhydrous conditions due to the presence of trace amounts of water in the confining medium (e.g., Yoshino et al. 2006; Dai and Karato 2009; Poe et al. 2010; Yang 2012; Yoshino and Katsura 2012). 
However, at one atmosphere, the hydrogen solubility in olivine is below $1 \mathrm{H} / 10^{6} \mathrm{Si}$, a level too small for water-related point defects to overcome the influence of electron holes and cation vacancies on electrical properties. Thus, the results from the Schock et al. (1989) study provide an ideal, anhydrous reference point.

Melt: Even a small amount of melt significantly enhances electrical conductivity of olivine-rich rocks, as long as the melt phase is interconnected (e.g., Roberts and Tyburczy 1999; Pommier et al. 2015b). In addition, shear deformation aligns melt pockets at the grain scale approximately parallel to the shear direction and can drive melt to segregate into melt-rich bands (Holtzman et al. 2003). Both grain-scale melt alignment and sample-scale segregation introduce electrical anisotropy. However, since melt does not form an interconnected network in our samples and is present in fewer than 1 in 10 triple junctions, its effect on electrical conductivity will be minimal (e.g., Sato and Ida 1984; Partzsch et al. 2000; Pommier et al. 2015b; Laumonier et al. 2017). Thus, conductivity results obtained on (melt-free) single crystals of olivine provide an ideal baseline for analyzing the results from measurements of electrical conductivity of polycrystalline samples.

Grain size and anisotropy: Conductivity measurements carried out on single crystals of olivine also provide an appropriate starting point for investigating the influence of grain size on electrical conductivity and of CPO on anisotropy in electrical conductivity in sheared polycrystalline samples. If there is no influence of grain size, the conductivity of dry, undeformed, melt-free olivine aggregates should be intermediate between the conductivities measured parallel to the 3 crystallographic axes in olivine crystals. 
In Figure $7 b$, we compare our results obtained on polycrystalline samples of San Carlos olivine with those published by Schock et al. (1989) for olivine single crystals studied at one atmosphere (anhydrous, melt-free conditions). These authors determined electrical conductivity along the three principal axes at controlled oxygen fugacity. We plot the Schock et al. (1989) results for oxygen fugacities corresponding to a molybdenum-molybdenum oxide (Mo/MoO) buffer, because our samples were in direct contact with molybdenum, which was likely lightly oxidized during our experiments. This choice is supported by the observation in Figure $1 \mathrm{~b}$ that conductivity is not affected by furnace type, namely, graphite versus rhenium, even though these two furnaces would produce relatively reducing and oxidizing conditions, respectively. We also normalized the Schock et al. (1989) data obtained at one atmosphere to a pressure of $2.8 \mathrm{GPa}$ using the activation volume of $0.6 \times 10^{-6}$ $\mathrm{m}^{3} / \mathrm{mol}$ reported by Xu et al. (2000) for comparison with our results. Correction for both oxygen fugacity (using O'Neill 1986) and pressure to the conditions of our experiments resulted in reduction of the Schock et al. (1989) conductivity data by a factor of $\sim 0.35$.

Prior results at room pressure by Roberts and Tyburczy $(1991 ; 1993)$ on grain boundary conductivity of olivine compacts and two different dunites at oxygen fugacities appropriate to the Mo/MoO oxygen buffer are shown in Figure $7 \mathrm{~b}$ for comparison, after extrapolation to a pressure of $2.8 \mathrm{GPa}$ using an activation volume of $0.6 \times 10^{-6} \mathrm{~m}^{3} / \mathrm{mol}$ from Xu et al. (2000). Their data are quite scattered but are largely consistent with the single crystal data from Schock et al. (1989) and our data for the compressional sample. Roberts and Tyburczy (1991) reported an activation energy 
of $238 \mathrm{~kJ} / \mathrm{mol}$ for grain boundary conductivity in polycrystalline olivine, which is similar to those for the single crystal data from Schock et al. (1989).

As is evident from Figure 7b, our conductivity results obtained on the sample that had been deformed in compression to low strain (PI1543) are only modestly more conductive than the data obtained on single crystals of San Carlos olivine (Schock et al. 1989) and on polycrystalline olivine by Roberts and Tyburczy (1991; 1993), after these data were corrected to the pressure and oxygen fugacities of our experiments. This observation suggests that electronic defects were the major charge carriers and that there were no major ionic contributions to conduction (as also noted by Roberts and Tyburczy 1993), such as might occur due to molybdenum from the electrodes (Watson 2002; Hayden and Watson 2006). It also indicates that our conductivity data were not significantly affected by trace concentrations of water or small amounts of melt. Since all our samples, both triaxially compressed and torsionally sheared, were prepared from the same batch of fine-grained powders of San Carlos olivine, the lack of any contribution of melt to the electrical conductivity of sample PI1543 indicates that melt cannot be responsible for the high electrical conductivity observed in our highly-sheared, fine-grained samples deformed in torsion.

Despite the similarity in conductivity, the activation enthalpy for conduction in PI1543 is significantly smaller than those for the single crystals of olivine or room pressure data on polycrystalline olivine (Figure $7 \mathrm{~b}$ ), as well as smaller than those measured for the torsional samples at comparable temperatures (Figure 7a,b). This result and the major differences in absolute conductivity suggest that the conduction 
mechanism for PI1543 may be fundamentally different from that in the torsion samples. The significant anisotropy of conductivity in PI1543 cannot be attributed to grain boundary effects, given the low strain and observation of no substantial difference in grain-boundary path lengths parallel to and normal to shear. One possible explanation for our PI1543 results may be that, with a grain size of about 15 $\mu \mathrm{m}$, electrical conductivity is dominated by the grain interiors, and that the presence of even a low concentration of water results in both increased conductivity and reduced activation enthalpy relative to the single crystal data. The anisotropy in conductivity for this sample would then reflect the weak CPO fabric induced due to compressional deformation.

With respect to the torsion samples, two questions arise: (i) Why is the electrical conductivity of the samples deformed in torsion so much higher than that of single crystals measured under anhydrous conditions? (ii) Why is the electrical conductivity anisotropic? The answers to both must be related to the contribution of grain boundary paths to electrical conductivity of our fine-grain, highly sheared polycrystalline olivine.

Indeed, electrical conductivity associated with transport along grain boundaries has been reported for fine-grained forsterite (Fe-free) samples (ten Grotenhuis et al. 2004). However, conduction in Fe-bearing olivine is dominated by electron holes, a mechanism not available in forsterite. Thus, a detailed comparison of results obtained on forsterite with those measured for San Carlos olivine is not warranted. 
The factor of $>10$ greater conductivity for the samples deformed in torsion compared to values reported for single crystals or observed in our coarser grained compressional sample is most directly explained by the difference in grain size. The grain size, $d$, of the samples deformed in torsion lies in the range 3 to $6 \mu \mathrm{m}$, while the grain size of the sample deformed in compression is $15 \mu \mathrm{m}$ (Table 1). Based on these grain size values, an increase in conductivity by a factor of 2.5 to 5 might be expected, if the contribution of grain boundaries to the bulk conductivity scales as $1 / d$ (Roberts and Tyburczy 1991). Thus, grain size can account for part, but not all, of the difference between the electrical conductivity of coarser and finer grained samples.

For the torsion samples, electrical anisotropy increases with shear strain (Figure $7 \mathrm{a}$ ) with highest conductivity parallel to the shear direction. In addition, as demonstrated by the crystallographic preferred orientation (CPO) maps (Figure 3), the [100] axes predominantly align parallel to the shear direction. Since conductivity in single crystals is largest parallel to [001] and smallest parallel to [100] and [010] (Figure 7b), the conductivity measurements in the sheared samples cannot have been controlled by grain interior processes. In addition, the sample with the greatest electrical anisotropy (PT0949) also has by far the strongest CPO (Figure 3). These observations indicate that, while crystallographic alignment does contribute to electrical anisotropy in the sheared samples, the overall behavior is dominated by grain-boundary conductivity. It is also striking that the finest grained sample (PT0264) with a grain size of $3.1 \mu \mathrm{m}$ is less conductive in the shear direction than the highest strained sample (PT0949) with a grain size of $6.4 \mu \mathrm{m}$. If grain size alone were important, the opposite should be true. These observations suggest not only that 
electrical conductivity along grain boundaries dominates the behavior of the sheared samples, but also that electrical conductivity of the grain boundaries is strongly misorientation dependent.

We therefore propose that, in addition to crystallographic alignment of the grain interiors, the development of a CPO also preferentially aligns grain boundaries that have high intrinsic conductivities in the direction of shear. With increasing strain, the strong CPO of the deformed samples is also characterized by a distinct grain boundary orientation distribution, as previously described by Marquardt et al. (2015) on synthetic and natural polycrystalline olivine. The grain boundaries aligned in the shear direction are crystallographically distinct from those aligned normal to the shear direction, resulting in marked variability in structure, energy, and transport properties. The grain boundaries along which electronic mobility is the highest are oriented such that the direction of highest conductivity is parallel to the shear direction. The existence of populations of grain boundaries in polycrystalline olivine with different transport properties is in agreement with first principles simulations of the structure of grain boundaries in Fo100 (Ghosh and Karki 2014). These simulations demonstrate that the detailed atomic and electronic structure of grain boundaries and thus their transport properties are controlled by the relative orientations of the adjoining grains. Our electrical conductivity and electrical anisotropy results support the conclusion that high-conductivity grain boundaries develop a strong orientation distribution linked to the measured CPO. The similarity in electrical conductivity along the direction normal to the shear plane (open symbols, Figure 7a) further suggests a complex interplay between grain size and CPO. 


\section{Concluding remarks}

Our electrical conductivity experiments demonstrate that grain boundaries become the dominant conduction paths at grain sizes less than $\sim 15 \mu \mathrm{m}$ for olivine. In addition, grain boundary conduction is anisotropic with transport in the shear direction as much as a factor of about 4 greater than transport normal to the shear plane. Within the shear plane, anisotropy is a factor of about 1.5. Thus, interpretation of electromagnetic data in lithospheric shear zones composed of mylonites and ultramylonites requires consideration of the effect of rock deformation on the bulk electrical response as well as the influence of water and/or melt. For coarser grainsize materials, such as occurs in mantle regions away from shear zones, one might expect that electrical conductivity would be dominated by the intragranular flux of electronic defects, as has been observed in previous experiments on natural San Quintin dunite by Roberts and Tyburczy (1993). 
Acknowledgements The authors wish to thank Bruce Watson for his thoughtful and insightful review of this manuscript. This study benefited from funding by the National Science Foundation through Cooperative Studies of the Earth's Deep Interior projects number EAR 14-61594 to the University of California San Diego and number EAR 15-20647 to the University of Minnesota. This research was also partially supported by the Consortium for Materials Properties Research in Earth Sciences under National Science Foundation Cooperative Agreement EAR 11-57758. Anne Pommier acknowledges support from the Alexander von Humboldt Foundation and thanks the Bayerisches GeoInstitut for hosting her during part of the study. Florian Heidelbach acknowledges support through Deutsche Forschungsgemeinschaft grant He3285/2-1. Stephen Mackwell acknowledges support from the National Aeronautics and Space Administration under CANNNX15AL12A. We are grateful to Jed Mosenfelder for his help with the FTIR, and Cameron Meyers for doing some EBSD measurements. We thank Katharina Marquardt for fruitful discussions, Mark Zimmerman for his help on the starting materials synthesis, Henrietta Cathey and Katherine Armstrong for assistance with microprobe and SEM analyses at Arizona State University and Bayerisches GeoInstitut, respectively, and Matej Pec for SEM analyses at the University of Minnesota. 


\section{References}

Ben Ismail WD, Mainprice D (1998) An olivine fabric database: an overview of upper mantle fabrics and seismic anisotropy. Tectonophys 296:145-157. doi:10.1016/S0040-1951(98)00141-3

Boneh Y, Wallis D, Hansen L, Krawczynski MJ, Skemer P (2017) Oriented grain growth and modification of 'frozen anisotropy' in the lithospheric mantle. Earth Planet Sci Lett 474:368-374. doi:10.1016/j.epsl.2017.06.050

Bystricky M, Kunze K, Burlini L, Burg J-P (2000) High shear strain of olivine aggregates: rheological, textural and seismic consequences. Science 290:15641567. doi:10.1126/science.290.5496.1564

Constable SC, Roberts JJ (1997) Simultaneous modeling of thermopower and electrical conduction in olivine. Phys Chem Miner 24:319-325

Dai L, Karato S-I (2009) Electrical conductivity of wadsleyite under high pressures and temperatures. Earth Planet Sci Lett 287:277-283. doi:10.1016/j.epsl.2009.08.012

Dai L, Li H, Hu H, Shan S (2008) Experimental study of grain boundary electrical conductivities of dry synthetic peridotite under high-temperature, highpressure, and different oxygen fugacity conditions. J Geophys Res 113:B12211. doi:10.1029/2008JB005820 
Dai L, Li H, Li C, Hu H, Shan S (2010) The electrical conductivity of dry polycrystalline olivine compacts at high temperatures and pressures. Miner Mag 74:849-857. doi:10.1180/minmag.2010.074.5.849

Dohmen R, Milke R (2010) Diffusion in polycrystalline materials: Grain boundaries, mathematical models, and experimental data. Rev Mineral Geochem 72:921-970. doi:10.2138/rmg.2010.72.21

Duba A, Constable S (1993) The electrical conductivity of a lherzolite. J Geophys Res 98:11885-11900

Du Frane WL, Roberts JJ, Toffelmier DA, Tyburczy JA (2005) Anisotropy of electrical conductivity in dry olivine. Geophys Res Lett 32: L24315. doi:10.1029/2005GL023879

Ghosh DB, Karki BB (2014) First principles simulations of the stability and structure of grain boundaries in $\mathrm{Mg}_{2} \mathrm{SiO}_{4}$ forsterite. Phys Chem Miner 41:163-171. doi:10.1007/s00269-014-0730-9

Hansen LN, Zimmerman ME, Kohlstedt DL (2011) Grain boundary sliding in San Carlos olivine: flow law parameters and crystallographic preferred orientation. J Geophys Res 116:B08201. doi:10.1029/2011JB008220

Hansen LN, Zimmerman ME, Kohlstedt DL (2012) The influence of microstructure on deformation of olivine in the grain-boundary sliding regime. J Geophys Res 117:B09201. doi:10.1029/2012JB009305

Hansen LN, Zhao Y-H, Zimmerman ME, Kohlstedt DL (2014) Protracted fabric 
evolution in olivine: implications for the relationship among strain, crystallographic fabric, and seismic anisotropy. Earth Planet Sci Lett 387:157168. doi:10.1016/j.epsl.2013.11.009

Hayden LA, Watson EB (2006) Grain boundary mobility of siderophile elements in MgO. Goldscmidt Conf Abs. doi:10.1016/j.gca.2006.06.480

Holtzman BK, Kohlstedt DL, Zimmerman ME, Heidelbach F, Hiraga T, Hustoft J (2003) Melt segregation and strain partitioning: implications for seismic anisotropy and mantle flow. Science 301:1227-1230. doi:10.1126/science.1087132

Huebner JS, Dillenburg RG (1995) Impedance spectra of hot, dry silicate minerals and rock: qualitative interpretation of spectra. Am Miner 80:46-64

Kohlstedt DL, Keppler H, Rubie DC (1996) Solubility of water in the $\alpha, \beta$ and $\gamma$ phases of $(\mathrm{Mg}, \mathrm{Fe})_{2} \mathrm{SiO}_{4}$. Contrib Miner Petrol 123:345-357

Laumonier M, Gaillard F, Muir D, Blundy J, Unsworth M (2017) Giant magmatic water reservoirs at mid-crustal depth inferred from electrical conductivity and the growth of the continental crust. Earth Planet Sci Lett 457:173-180. doi:10.1016/j.epsl.2016.10.023

Marquardt K, Rohrer GS, Morales L, Rybacki E, Marquardt H, Lin B (2015) The most frequent interfaces in olivine aggregates: the GBCD and its importance for grain boundary related processes. Contrib Miner Petrol 170:40. doi:10.1007/s00410015-1193-9 


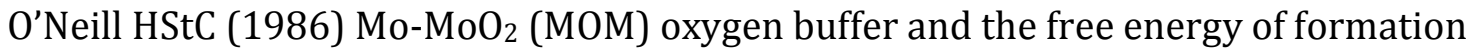
of $\mathrm{MoO}_{2}$. Am Miner 71:1007-1010

Paterson MS (1982) The determination of hydroxyl by infrared absorption in quartz, silicate glasses and similar materials. Bull Miner 105:20-29

Poe BT, Romano C, Nestola F, Smyth JR (2010) Electrical conductivity anisotropy of dry and hydrous olivine at 8 GPa. Phys Earth Planet Inter 181:103-111. doi:10.1016/j.pepi.2010.05.003

Pommier A, Leinenweber K, Kohlstedt DL, Qi C, Garnero EJ, Mackwell SJ, Tyburczy JA (2015a) Experimental constraints on the electrical anisotropy of the lithosphere-asthenosphere system. $\quad$ Nature 522:202-206. doi:10.1038/nature14502

Pommier A, Leinenweber K, Tasaka M (2015b) Experimental investigation of the electrical behavior of olivine during partial melting under pressure and application to the lunar mantle. Earth Planet Sci Lett 425:242-255. doi:10.1016/j.epsl.2015.05.052

Roberts JJ, Tyburczy JA (1991) Frequency dependent electrical properties of polycrystalline olivine compacts. J Geophys Res 96:16,205-16,222. doi:10.1029/91JB01574

Roberts JJ, Tyburczy JA (1993) Frequency dependent electrical properties of dunite as functions of temperature and oxygen fugacity. Phys Chem Miner 19:545-561

Roberts JJ, Tyburczy JA (1999) Partial-melt electrical conductivity: influence of melt 
composition. J Geophys Res 104:7055-7065. doi:10.1029/1998JB900111

Sato H, Ida Y (1984) Low frequency electrical impedance of partially molten gabbro: the effect of melt geometry on electrical properties. Tectonophys 107:105-134

Schock RN, Duba A, Shankland TJ (1989) Electrical conduction in olivine. J Geophys Res 94:5829-5839

ten Grotenhuis SM, Drury MR, Peach CJ, Spiers CJ (2004) Electrical properties of finegrained olivine: evidence for grain boundary transport. J Geophys Res 109:B06203. doi:10.1029/2003JB002799

Tommasi A, Mainprice D, Canova G, Chastel Y (2000) Viscoplastic self-consistent and equilibrium-based modeling of olivine lattice preferred orientations: implications for the upper mantle seismic anisotropy. J Geophys Res 105:78937908. doi:10.1029/1999JB900411

Underwood E (1970) Quantitative Stereology. Addison-Wesley, Reading, MA

Wagner J, Adjaoud O, Marquardt K, Jahn S (2016) Anisotropy of self-diffusion in forsterite grain boundaries derived from molecular dynamics simulations. Contrib Miner Petrol 171:98. doi:10.1007/s00410-016-1308-y

Watson EB (2002) Mobility of siderophile elements in grain boundaries of periclase and periclase/olivine aggregates. EOS Trans AGU 83 Spring Meet Suppl Abs V52B-03

Xu YS, Shankland TJ, Duba A (2000) Pressure effect on electrical conductivity of 
mantle olivine. Phys Earth Planet Inter 118:149-161. doi:10.1016/S00319201(99)00135-1

Yang X (2012) Orientation-related electrical conductivity of hydrous olivine, clinopyroxene and plagioclase and implications for the structure of the lower continental crust and uppermost mantle. Earth Planet Sci Lett 317-318:241-250. doi:10.1016/j.epsl.2011.11.011

Yoshino T, Matsuzaki T, Yamashita S, Katsura T (2006) Hydrous olivine unable to account for conductivity anomaly at the top of the asthenosphere. Nature 443:973-976. doi:10.1038/nature05223

Yoshino T (2010) Laboratory electrical conductivity measurement of mantle minerals. Surv Geophys 31:163-206. doi:10.1007/s10712-009-9084-0

Yoshino T, Katsura T (2012) Re-evaluation of electrical conductivity of anhydrous and hydrous wadsleyite. Earth Planet Sci Lett 337-338:56-67. doi:10.1016/j.epsl.2012.05.023

Zhang B, Yoshino T, Yamazaki D, Manthilake G, Katsura T (2014) Electrical conductivity anisotropy in partially molten peridotite under shear deformation. Earth Planet Sci Lett 405:98-109. doi:10.1016/j.epsl.2014.08.018 


\section{Figure captions:}

Fig. 1 Experimental protocol. a) Cross-section of the electrical conductivity cell composed of a 14/8 multi-anvil assembly with a corner-truncation edge length of 8 $\mathrm{mm}$ and a pressure-media edge length of $14 \mathrm{~mm}$ ). Both electrodes are made of $\mathrm{W}-\mathrm{Re}$ thermocouple wire with one electrode also serving as a thermocouple. b) Electrical conductivity vs. temperature of sample PT0941 demonstrating experimental reproducibility. One experiment was conducted using a graphite furnace and the other using a rhenium furnace. In both cases, essentially identical conductivity data were obtained at defined T. c) Example of complex impedance spectra obtained at three different temperatures (real part versus imaginary part of complex impedance (Z) for a sheared sample of olivine (PT0949) at $2.8 \mathrm{GPa}$. Each dot corresponds to a single frequency

Fig. 2 Starting materials. a) EBSD maps of the starting materials, showing the crystallographic orientations of the grains and higher grain boundary density (i.e., smaller grain size) in the torsionally sheared materials than in the low-strain compression sample (PI1543). Coloring as follows: grains with [100] parallel to the shear direction are in red, grains with [010] parallel to the shear direction are in yellow-green, and grains with [001] parallel to the shear direction are in blue. The sense of shear is represented by the black arrows. b) Pole figure plot for sample PT0941 with the matching coloring as in a), illustrating the orientations of the grains relative to the shear direction 
Fig. 3 Pole figures showing the crystal-preferred orientation (CPO) of the four starting materials after deformation but prior to electrical conductivity measurements. The top set of figures shows the slightly compressed $(\varepsilon \sim 0.2)$ sample (PI1543), while the lower 3 sets show the torsionally sheared samples with increasing shear strain. Pole figures are presented in the lower hemisphere using an equal area projection. Black arrows indicate the sense of deformation

Fig. 4 Back-scattered electron images of the three sheared samples after electrical measurements, showing that a) there is no significant amount of melt $(<0.1$ vol. $\%)$, and b, c, d) the initial deformation texture (see Figure 2) was preserved during the experiment in the multi-anvil apparatus. The sense of shear is represented by the red arrows

Fig. 5 Pole figures showing the crystal-preferred orientation (CPO) of two samples retrieved after electrical conductivity experiments PT0941-1 and PT0949-2. Pole figures are presented in the lower hemisphere using an equal area projection. The sense of shear is represented by the black arrows

Fig. 6 FTIR analyses. a) FTIR spectra of two sheared samples after the electrical experiments (PT0941-1 and PT0949-2). For each sample, the water concentration is $<150$ ppm H/Si. b) Comparison of the FTIR spectra in a) with analyses on water saturated olivine at $3 \mathrm{GPa}$ from Kohlstedt et al. (1996) (containing 10,000 ppm 
H/Si). Hydrogen concentration was determined using the Paterson (1982) calibration and correcting by a factor of 3.5 (Bell et al. 2003)

Fig. 7 Electrical conductivity results. a) Electrical conductivity - temperature plot showing experimental results for polycrystalline olivine samples deformed to a small strain in compression (PI1543) and to large strains in torsional shear (PT0941, PT0264 and PT0949).. The data were collected at a pressure of $2.8 \mathrm{GPa}$ and with the oxygen fugacity fixed by the Mo/MoO buffer. Open, filled and + symbols represent data perpendicular to the shear plane (orientation 1), parallel to the shear direction (orientation 2), and in the shear plane perpendicular to the shear direction (orientation 3), respectively. Experimental data for sample PI1543 deformed in compression and for sample PT0264 measured parallel to the shear direction are from Pommier et al. (2015a). Dashed (orientation 1), solid (orientation 2) and dotdashed (orientation 3) lines correspond to Arrhenius fits to the data for $\mathrm{T}>850^{\circ} \mathrm{C}$ (Table 2). b) Comparison of experimental results at high temperature (line colors and styles are the same as in Figure 7a) with olivine (Fo90) single crystal electrical conductivity data from Schock et al. (1989) parallel to the three low-index olivine crystallographic axes. The single crystal data, which were collected at room pressure and oxygen fugacity set by a constant $10: 1$ mixture of $\mathrm{CO}_{2}: \mathrm{CO}$, were corrected to a pressure of $2.8 \mathrm{GPa}$ and oxygen fugacity set by the Mo/MoO buffer, as noted in the text. Also shown (+) are data from Roberts and Tyburczy $(1991 ; 1993)$ for samples measured at room pressure at oxygen fugacities equivalent to the Mo/MoO buffer. These data were also corrected to a pressure of $2.8 \mathrm{GPa}$ 